\title{
MELISSA: Laser Ion Source Setup at CERN-MEDICIS facility. Blueprint
}

\author{
V. M. Gadelshin ${ }^{1,6}$, V. Barozier ${ }^{2}$, T. E. Cocolios 3 , V. N. Fedosseev², R. Formento-Cavaier ${ }^{4,5}$, F. Haddad ${ }^{4}$, \\ B. Marsh ${ }^{2}$, S. Marzari ${ }^{2}$, S. Rothe ${ }^{2}$, T. Stora ${ }^{2}$, D. Studer ${ }^{1}$, F. Weber ${ }^{1}$, and K. Wendt ${ }^{1}$ \\ ${ }^{1}$ Institute of Physics, Johannes Gutenberg University Mainz, Staudingerweg 7, 55128 Mainz, Germany \\ 2 Engineering Department, CERN, 1 Esplanade des Particules, 1211 Genève, Switzerland \\ ${ }^{3}$ Institute for Nuclear and Radiation Physics, KU Leuven, Celestijnenlaan 200D, 3001 Heverlee, Belgium \\ ${ }^{4}$ GIP Arronax, 1 rue Aronnax, 44800 Saint Herblain, France \\ ${ }^{5}$ Advanced Accelerator Applications, A Novartis company, 20 rue Diesel, 01630 Saint-Genis-Pouilly, France \\ ${ }^{6}$ Ural Federal University, Mira st. 19, 620002 Ekaterinburg, Russia
}

\section{A B S T R A C T}

The Resonance Ionization Laser Ion Source (RILIS) has become an essential feature of many radioactive ion beam facilities worldwide since it offers an unmatched combination of efficiency and selectivity in the production of ion beams of many different chemical elements. In 2019, the laser ion source setup MELISSA is going to be established at the CERN-MEDICIS facility, based on the experience of the workgroup LARISSA of the University Mainz and CERN ISOLDE RILIS team. The purpose is to enhance the capability of the radioactive ion beam supply for end users by optimizing the yield and the purity of the final product. In this article, the blueprint of the laser ion source, as well as the key aspects of its development and operation are presented.

Keywords: CERN-MEDICIS, isotope separation, laser resonance ionization, titanium:sapphire, lanthanides, MELISSA, nuclear medicine.

\section{Introduction}

The CERN-MEDICIS radioactive ion beam facility aims for the production of innovative medical radioisotopes [1]. It is foreseen to use two production routes. The first one implies the use of the $1.4 \mathrm{GeV}$ proton beam coming from the CERN Proton-Synchrotron for irradiation of the target material at one of the ISOLDE target stations, with subsequent radionuclide extraction at the dedicated CERN-MEDICIS mass separator. The targets are transferred to the separator bunker from their irradiation point using the specially developed mono rail system «MONTRAC». The "off-line" nature of the extraction process almost excludes a possibility to produce radionuclides with a half-life less than minutes, but the transfer time of the target unit to the mass separator is short enough for a regular production of isotopes having a half-life above one hour, which corresponds to most radioisotopes of medical interest.

The second production route is based on the radionuclide extraction from targets pre-irradiated and provided by external institutions, such as nuclear reactors, medical accelerators and cyclotrons, and nuclear waste depositaries. This additional operation mode of MEDICIS is particularly important during the periods of unavailability of the CERN accelerator complex, thereby enabling a radionuclide supply to the user even during the CERN long-shutdown maintenance periods, expanding capabilities in production of useful radioisotopes [2].

The MEDICIS mass separator uses a conventional dipole electromagnet for mass selection, which requires a work substance to be in the form of a mono-energetic ion beam. Traditionally, surface ionization or plasma ionization methods are used, and all ions are extracted at the same energy (30-60 keV). However, these ion sources offer limited selectivity, and thus usually give rise to undesired isobaric contamination. For many areas of radionuclide applications, the chemical and isotopic purity is of highest importance. The presence of significant or dominant isobaric or isotopic impurities is particularly not acceptable for use in the personalized nuclear medicine. The impurities not only reduce the effectiveness of a treatment, but may cause unintended irradiation as well as a contamination of healthy tissues of a living being with other radionuclides. Almost independent of the production mechanism, achieving the required purity requires various individual steps, often including element-specific radiochemistry, procedure that can significantly increase the total production time, leading to a reduction in the specific activity of short-lived species.

Today the resonance ionization laser ion source (RILIS) technique is successfully used at the majority of radioactive ion beam facilities worldwide for efficient and selective ionization of a work substance [3]. The working principle is multi-step resonance photo- excitation and subsequent ionization of atoms of the desired chemical element. The combination of the conventional electromagnetic mass separation and the laser resonance ionization allows to extract selectively ion beams of a desired radionuclide. Moreover, the laser ion source achieves a high ionization efficiency (typically more than 10\%) for numerous elements, comparable, if not superior, to that of the plasma ionization approach.

A laser ion source for the MEDICIS facility was therefore foreseen as a future advance of MEDICIS during the initial design and construction of the facility. As a core of the future setup, an allsolid-state laser system was chosen, jointly designed with the Johannes Gutenberg University Mainz. It is based on solid-state titanium:sapphire lasers, which have proven to be sufficiently reliable and flexible for continuous operation at ISOL facilities. The layout, configuration and current status of the MEDICIS Laser Ion Source Setup At CERN, named MELISSA, is described here.

\section{MEDICIS Laser Ion Source Setup - MELISSA}

\subsection{Laser laboratory design}

Since CERN-MEDICIS is a radioactive facility, the laboratory equipment is contained within restricted areas, which must fulfill special requirements in accordance with the radiation safety regulation. To allow access to and operation of the laser laboratory at any time, and to avoid unnecessary dose uptake for the laser operators, it was decided to build a room, separated from the radiochemical laboratory and the mass separator bunker.

Fig. 1 illustrates a schematic of the upcoming laser laboratory. It is divided into three rooms: a dressing room (buffer laser-safe entrance area); the main room with the optical tables, where the laser system is located; a supplementary room for closed-circuit 


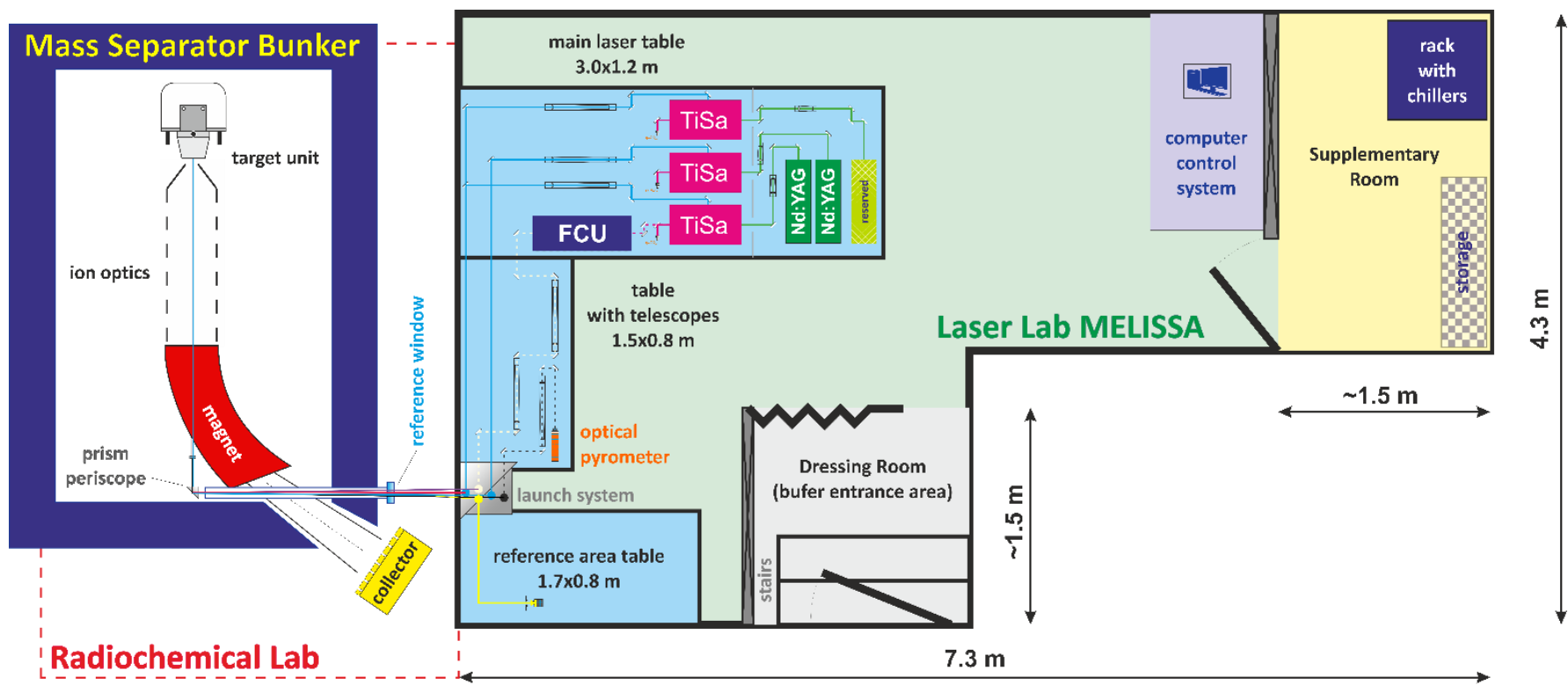

Fig. 1. Overview of the MELISSA laser laboratory and the MEDICIS mass separator layout.

laser chillers, to minimize the heat emission and noise stress into the main laser laboratory. The lasers in the room are interlocked against non-granted access, and the laboratory is physically isolated from other MEDICIS environments. A dedicated air conditioning and ventilation system was installed to guarantee a stable performance of the laser. At the point of the laser beam launch system towards the separator, lasers will pass through a window, which eliminates air flow between the laboratory and the radiationcontrolled areas. The laser beam path from the laser laboratory to the separator bunker is enclosed to avoid any laser hazards for MEDICIS operators or users. Therefore, the laser laboratory is the only area where laser hazards exist during normal operation.

The optical path distance from the laser laboratory to the target unit is 13 meters. As the separator bunker is a radioactive area, and therefore inaccessible during normal operation mode, a method of the reliable overlap of laser beams in the ion source was established, without the need to open up the laser shielding or physically access the target bunker. A pair of reference beams are produced by the Fresnel reflections from each surface of the wedged ( 3 arcmin) reference window (Fig. 1), located at $\sim 8 \mathrm{~m}$ distance from the target. These will be directed back to the laser laboratory to be distributed in a dedicated 'virtual ion source' reference area. At this location, laser beam monitoring and stabilization systems, similar to one developed at the ISOLDE-RILIS [4], will be established. The laser path to the target unit can be also used to observe and control the thermal behavior in the ion source during the extraction process via an optical pyrometer, installed in the laser laboratory. Eventually, the MELISSA installation will be integrated into the control system of the MEDICIS facility, with a long-term goal of becoming a «turn-key»system, where lasers will be automatically tuned to required wavelengths and aligned into the ion source in accordance with greatly reduced laser-operator dependence compared to that of the current ISOLDE-RILIS system.

\subsection{Laser system development}

To minimize the laser setup, operation and maintenance time, which introduce some constraints on the production planning, it is foreseen for MELISSA to use highly reliable wide-range tunable pulsed titanium:sapphire lasers constructed by the LARISSA group of the University Mainz, pumped by commercial frequency doubled
Nd:YAG lasers. The use of the all-solid-state laser system instead of dye lasers allows performing the ionization and collection of radioisotopes without interruptions for changing of dye solutions. It also prevents handling of chemically hazardous substances by laser operators, and simplifies safety requirements for the laser laboratory.

As can be seen from Fig. 1, each titanium:sapphire laser will be pumped by a dedicated Nd:YAG laser. A multi-channel pulse delay generator will trigger the pump lasers, and enable optimization of the laser pulse synchronization with nanosecond precision, what is essential for achieving the maximum ionization efficiency. Pumped with $15 \mathrm{~W}$ at $532 \mathrm{~nm}$ with a repetition rate of $10 \mathrm{kHz}$, the titanium:sapphire laser can emit up to $5 \mathrm{~W}$ output power using the standard "Z" cavity, with a spectral linewidth and pulse length of approximately $5 \mathrm{GHz}$ and $40 \mathrm{~ns}$, respectively [5]. The fundamental emission range spans from 690 to $960 \mathrm{~nm}$. The frequency conversion (FCU) of laser radiation to second, third, or fourth harmonics (SHG, THG, FHG), passing the laser beam through nonlinear crystals (bismuth barium borate, $\mathrm{BiBO}$, or beta-barium borate, $\mathrm{BBO}$ ), extends the wavelength tuning range to the blue and UV regions of the optical spectrum.

To reduce the time required for changing the ionization scheme, the most advanced automated grating-tuned titanium:sapphire laser with intra-cavity SHG (Fig. 2) was developed [6]. Principally, the intra-cavity SHG with a single non-linear crystal can cover a wavelength range i.e. from $375 \mathrm{~nm}$ to $475 \mathrm{~nm}$. The output power with up to $1.5 \mathrm{~W}$ is lower, compared to the standard cavity, but still high enough for ionization application: as shown in [7], ionization schemes using transitions in this wavelength range could be identified with saturation threshold typically below $100 \mathrm{~mW}$. Operated by a dedicated software, controlling the grating and nonlinear crystal angles in relation to output wavelength and power, this laser allows a rapid and automated switching between required wavelengths.

\subsection{Laser resonance ionization spectroscopy}

Today laser resonance ionization schemes almost for all metallic elements are available in literature [3], [8], [9]. Because of the historical development of laser technologies, the majority of known schemes are optimized to the emission range of various dye 
lasers. The use of titanium:sapphire lasers requires additional investigation of atomic spectra of elements, if the existing transitions are not in their working range.

The characterization of a suitable multi-step laser resonance ionization scheme for a desired element is necessary to achieve the optimal ionization efficiency along with a sufficient chemical and isotopic purity, and reliability of the production process. Therefore, for chemical elements of interest to MEDICIS [1], laser resonance ionization spectroscopy studies were undertaken to define laser ionization schemes, suitable for the emission range of titanium:sapphire lasers. This work is focused on rare-earth elements, because many medically relevant isotopes, being promising for the theranostics approach [10], are found within this group. Several lanthanides, such as $\mathrm{Dy}, \mathrm{Ho}$, and $\mathrm{Yb}$, were already investigated [11], [12]. Results on Lu are published in [7]. New results on $\mathrm{Er}$ [2], $\mathrm{Tb}$ and $\mathrm{Nd}$ are currently evaluated, and will be reported in forthcoming publications.

It was observed that many lanthanides can be easily and efficiently ionized by two-step excitation schemes with transitions in the blue spectral range $(350-475 \mathrm{~nm})$. With the use of two automated grating-tuned titanium:sapphire lasers with intra-cavity SHG, a switching-time of less than a minute between different ionization schemes has been demonstrated. This feature is desirable for fast extraction of different short-lived radionuclides from an irradiated target, expanding the variety of producing radioisotopes.

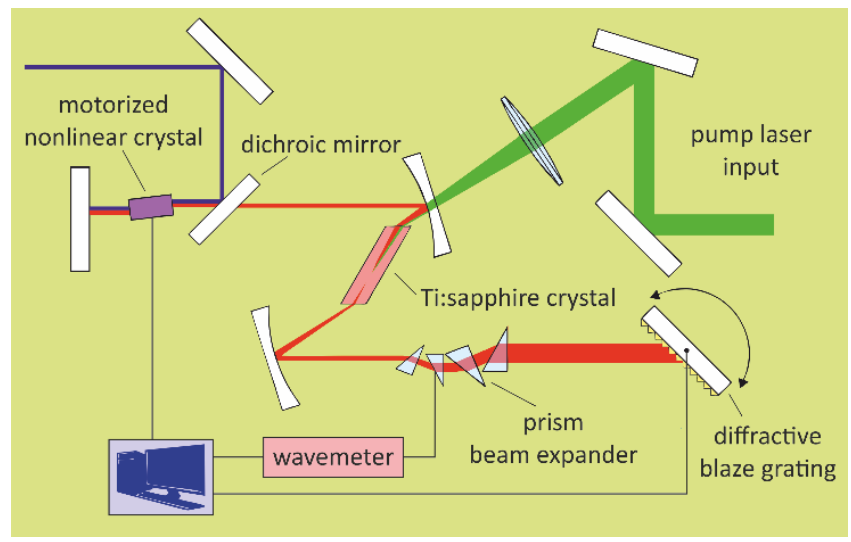

Fig. 2. Schematic layout of an automated grating tuned titanium:sapphire laser with intracavity frequency doubling

\section{Conclusions and outlook}

In 2019, CERN-MEDICIS will install and commission the laser ion source setup MELISSA. The use of element selective ionization will improve the ion beam purity of extracted radioisotopes. Several laser resonance excitation schemes for lanthanides of medical interest have already been developed with efficiencies more than $50 \%$, measured at the Mainz RISIKO mass separator. These schemes will be used to produce at the MEDICIS facility the first laser ionized samples of medical radionuclides (approx. in April, 2019), and will be reported in the upcoming $\mathrm{PhD}$ theses of Vadim Gadelshin and Roberto Formento-Cavaier.

Because of the second long shutdown of the CERN accelerator complex (LS2), which will continue until 2021, MEDICIS will only be supplied with externally irradiated targets. Their place of origin and subsequent operation may be quite different from those of the CERN on-site irradiation. Externally irradiated targets may arrive in a variety of chemical forms and conditions. Thus, the ion source chemistry and physics need to be specially investigated to achieve the best production results in each individual case.
The radionuclide extraction from externally irradiated targets, e.g. for $\mathrm{Tb}$ radioisotope production by proton irradiation of gadolinium targets, can be accompanied by a strong isobaric contamination on the desired mass. The implementation of the laser ion source may enable isotope extraction at a lower temperature, decreasing the undesired surface ionization of contaminants. Optimizing the configuration of the ion source and the design of the target unit, as well as the operational procedure, isobaric contaminants can be additionally suppressed, leading to a high purity of the products.

It is also necessary to continue the development work on extension of the wavelength range available from the titanium:sapphire lasers, as well as on enhancement of the output power; towards the design of a "turn-key" laser system, aiming for an automatically aligned and optimized laser system for a predefined ionization scheme of a desired element. By achieving a higher efficiency and enabling the direct ionization of a required isotope, rather than waiting for the decay of short- or long-lived contaminants, the overall production time will be reduced in many cases. It will enlarge the variety of producible medical radionuclides, delivering with a high specific activity.

\section{Acknowledgements}

This research project has been supported by a Marie Skłodowska-Curie Innovative Training Network Fellowship of the European Commission's Horizon 2020 Programme under contract number 642889 MEDICIS-PROMED; by a Research and Innovation Programme of the European Commission's Horizon 2020 under contract number 654002 (ENSAR2-RESIST); by a grant from the French National Agency for Research called "Investissements d'Avenir", Equipex Arronax-Plus no. ANR-11EQPX-0004 and Labex IRON no. ANR-11-LABX-18-01; by FWO-Vlaanderen (Belgium), and by a KU Leuven START Grant; by the German Federal Ministry of Education and Research under the consecutive projects 05P12UMCIA and 05P15UMCIA.

\section{References}

[1] dos Santos Augusto R. M. et al.: CERN-MEDICIS (Medical Isotopes Collected from ISOLDE): a new facility. Applied Sciences, 4, 265 (2014).

[2] Formento-Cavaier R. et al.: Very high specific activity erbium Er-169 production for potential receptor targeted radiotherapy. In this proceedings, (2019).

[3] Fedosseev V. et al.: Ion beam production and study of radioactive isotopes with the laser ion source at ISOLDE. Journal of Physics G: Nuclear and Particle Physics, 44(8), 084006 (2017).

[4] Rothe S. et al.: Laser ion beam production at CERN-ISOLDE: New features - More possibilities. Nuclear Instruments and Methods in Physics Research B, 376, 91 (2016).

[5] Rothe S. et al.: A complementary laser system for ISOLDE RILIS. Journal of Physics: Conference Series, 312, 052020 (2011).

[6] Weber F. Effizienter elementselektiver Nachweis von Lanthaniden mit RIMS. Master thesis, Johannes Gutenberg University Mainz, 2018.

[7] Gadelshin V.M. et al.: Laser resonance ionization spectroscopy on lutetium for the MEDICIS project. Hyperfine Interactions, 238:28 (2017)

[8] Cross-facility database on laser ionization schemes for chemical elements, [Online]. Available: http://riliselements.web.cern.ch/riliselements/index.php [2018, December]. CERN, Geneva.

[9] Fedosseev V.N. et al.: Resonance laser ionization of atoms for nuclear physics. Physica Scripta, 85, 058104 (2012).

[10] Tishchenko V. K. et al.: Radiopharmaceuticals based on polyaminophosphonic acids labeled with $\alpha$-, $\beta$-, and $\gamma$ emitting radionuclides (Review). Pharmaceutical Chemistry Journal, 49(7), 3 (2015).

[11] Studer D. et al.: Resonance ionization spectroscopy in dysprosium. Excitation scheme development and re-determination of the first ionization potential. Hyperfine Interactions, 238:8 (2017).

[12] Schneider F. et al.: Resonance ionization of holmium for ion implantation in microcalorimeters. Nuclear Instruments and Methods in Physics Research B, 376, 388 (2016). 